\title{
Joint Multipath-Doppler Diversity in Mobile Wireless Communications
}

\author{
Akbar M. Sayeed, Member, IEEE, and Behnaam Aazhang, Senior Member, IEEE
}

\begin{abstract}
We introduce a new approach for achieving diversity in spread-spectrum communications over fast-fading multipath channels. The RAKE receiver used in existing systems suffers from significant performance degradation due to the rapid channel variations encountered under fast fading. We show that the Doppler spread induced by temporal channel variations in fact provides another means for diversity that can be further exploited to combat fading. We develop the concept of Doppler diversity and propose a framework that exploits joint multipathDoppler diversity in an optimal fashion. Performance analysis shows that even the relatively small Doppler spreads encountered in practice can be leveraged into significant diversity gains via our approach. The framework is applicable in several mobile wireless multiple access systems and can provide substantial performance improvement over existing systems.
\end{abstract}

Index Terms-Diversity, Doppler, fast fading, multipath, timefrequency RAKE.

\section{INTRODUCTION}

$\mathbf{S}$ IGNAL fading caused by the channel is a major factor limiting the performance of mobile wireless communication systems. The receiver performance is significantly degraded by the fluctuations in received signal power caused by fading. Diversity techniques, which essentially amount to transmitting the signal over multiple independent fading channels, are used in practice to combat fading [1]. The mobile wireless channel affords inherent diversity that can be exploited with appropriate signaling and reception. The RAKE receiver used in code-division multiple access (CDMA) systems utilizes the large bandwidth of spread-spectrum signaling waveforms to achieve multipath diversity [1]. The RAKE receiver is optimal under, and provides a substantial guard against, slow fading, in which the channel characteristics vary slowly over time [1].

However, the increased mobility of users in cellular communications often results in fast fading in which the channel exhibits rapid temporal variations [1]-[3]. The resulting Doppler spread induced in the signal substantially degrades the performance of the RAKE receiver due to errors in channel state estimation [3]-[6]. In fact, existing systems

Paper approved by C. Robertson, the Editor for Spread Spectrum Systems of the IEEE Communications Society. Manuscript received May 28, 1997; revised March 3, 1998 and June 29, 1998. This work was presented in part at the 1997 Conference on Information Sciences and Systems, Baltimore, MD. The research was supported by the NSF and the Texas Advanced Technology Program.

A. M. Sayeed is with the Department of Electrical and Computer Engineering, University of Wisconsin-Madison, Madison, WI 53706 USA (e-mail: akbar@engr.wisc.edu).

B. Aazhang is with the Department of Electrical and Computer Engineering, Rice University, Houston, TX 77005 USA (e-mail: aaz@ rice.edu).

Publisher Item Identifier S 0090-6778(99)00790-4. exhibit a limiting bit-error probability (BEP) floor under such conditions that cannot be improved by increasing the transmitted signal power [3], [5]. Current approaches for counteracting the degradation of the RAKE receiver aim at making the receiver robust to Doppler effects. For instance, the noncoherent RAKE receiver [1] is often used in practice to eliminate the need for channel estimation, thereby incurring an inherent loss of 3-6 dB in signal-to-noise ratio (SNR). Other approaches modify the channel estimation procedure in an ad hoc manner to contain the degradation [4]-[6]. However, all existing approaches for salvaging the performance of the RAKE receiver are suboptimal and, more importantly, ignore vital channel characteristics that offer the potential for substantial gains via additional diversity.

We propose a new CDMA framework in this paper that is directly inspired by the characteristics of the fast fading channel and fully exploits its inherent diversity. The key idea behind our approach is joint multipath-Doppler diversity: exploiting the cause of degradation in existing systems-Doppler effects-to provide another means for diversity, in conjunction with multipath diversity. We introduce the concept of Doppler diversity and develop a framework that exploits joint multipath-Doppler diversity in an optimal manner. As we demonstrate in this paper, joint multipath-Doppler diversity promises remarkable performance gains-even the relatively small Doppler spreads encountered in practice can yield significant diversity gains via appropriate signaling and reception.

The fast fading channel is an inherently time-varying system, and our methodology employs joint time-frequency representations (TFR's), which are powerful tools for timevarying signal processing [7], [8]. The workhorse of our framework is a canonical time-frequency-based decomposition of the mobile wireless channel into a series of independent fading channels. The decomposition naturally leads to a time-frequency generalization of the RAKE receiver that exploits joint multipath-Doppler diversity. We consider both coherent and noncoherent signaling and derive optimal TFRbased receiver structures that admit efficient realizations via a bank of conventional RAKE receivers. The framework is applicable in several multiple access mobile wireless systems and promises substantially improved performance due to its inherently higher level of diversity.

We introduce relevant concepts about TFR's in the next section and provide a time-frequency description of the timevarying channel. The framework for joint multipath-Doppler diversity is developed in Section III. In Section IV, we demonstrate the performance gains achievable with our approach and 
provide a discussion of practical system modalities which can maximally benefit from it. Concluding remarks are provided in Section V.

\section{PRELIMINARIES}

\section{A. Time-Frequency Representations}

TFR's are powerful tools for representing time-varying signals and systems. They are two-dimensional signal representations jointly parameterized by both time and frequency. As we will see, TFR's are ideally suited for processing signals transmitted over the inherently time-varying mobile wireless channel.

The concepts of time- and frequency-shifts play a fundamental role in the theory of TFR's, and we will use them in our development. We denote time- and frequency-shifts by operators $\mathbf{T}_{\tau}$ and $\mathbf{F}_{\theta}$, respectively, defined as

$$
\left(\mathbf{T}_{\tau} s\right)(t) \stackrel{\text { def }}{=} s(t-\tau), \quad\left(\mathbf{F}_{\theta} s\right)(t) \stackrel{\text { def }}{=} s(t) e^{j 2 \pi \theta t}
$$

where the parameter $\tau$ denotes the value of the time-shift, and $\theta$ denotes the value of the frequency-shift introduced in the signal.

The TFR of interest to us in this paper is the short-time Fourier transform (STFT) which is defined for a signal $s(t)$ as

$$
\operatorname{STFT}_{s}^{(g)}(\theta, \tau) \stackrel{\text { def }}{=} \int s(t) g^{*}(t-\tau) e^{-j 2 \pi \theta t} d t
$$

for a given "window" function $g(t)$. It is the projection of the signal onto a family of functions $\psi_{(\theta, \tau)}(t)$ that are time- and frequency-shifted versions of $g(t)$

$$
\begin{aligned}
& \operatorname{STFT}_{s}^{(g)}(\theta, \tau) \stackrel{\text { def }}{=}\left\langle s, \psi_{(\theta, \tau)}\right\rangle \\
& \psi_{(\theta, \tau)}(t) \stackrel{\text { def }}{=}\left(\mathbf{F}_{\theta} \mathbf{T}_{\tau} g\right)(t)=g(t-\tau) e^{j 2 \pi \theta t}
\end{aligned}
$$

where $\langle\cdot, \cdot\rangle$ denotes the inner product. The STFT can also be interpreted as a time-frequency correlation function. In fact, $\operatorname{STFT}_{s}^{(g)}(\theta, \tau)$ is the correlator output for optimally detecting the signal $\psi_{(\theta, \tau)}(t)$ in the presence of additive white Gaussian noise (AWGN).

\section{B. Channel and Signal Models}

We next develop the time-frequency-based channel and signal models that underlie the development in this paper. Our discussion is in the context of CDMA systems, and since the focus of this paper is on the effects of the channel, we restrict our discussion to the single-user case, binary signaling, negligible intersymbol interference, and perfect synchronization.

The baseband transmitted signal $x(t)$ can be represented as

$$
x(t)=\sum_{p} q_{p}(t-p T)
$$

where $T$ is the symbol period, $q_{p}(t)$ is the information bearing waveform, $q_{p}(t) \in\left\{q^{1}(t), q^{0}(t)\right\}$, that could either correspond to antipodal signaling $\left(q^{1}(t)=-q^{0}(t)\right)$ or orthogonal

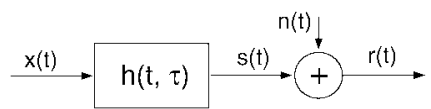

Fig. 1. Mobile wireless channel: linear time-varying system.

signaling $\left(\left\langle q^{1}, q^{0}\right\rangle=0\right)$. The spread-spectrum waveforms $q^{m}(t), m=0,1$ take the form

$$
q^{m}(t)=\sum_{n=0}^{P-1} c^{m}[n] v\left(t-n T_{c}\right), \quad 0 \leq t<T
$$

where $v(t)=I_{\left[0, T_{c}\right)}(t)$, the chip waveform, $T_{c}$ is the chip interval, $I_{[a, b)}(t)$ is the indicator function of $[a, b), T=P T_{c}$, and $c^{m}[n]$ is the spreading code corresponding to $q^{m}(t)$ [1]. The signal bandwidth $B \approx 1 / T_{c}$, and the parameter $P=T / T_{c} \approx T B \gg 1$ is called the spreading gain of the spread-spectrum system.

As illustrated in Fig. 1, the baseband signal $r(t)$ at the receiver is given by

$$
r(t)=s(t)+n(t)=\int_{0}^{\infty} h(t, \tau) x(t-\tau) d \tau+n(t)
$$

where the channel is described by the kernel $h(t, \tau)$ which corresponds to a time-varying linear system [1], [9], and $n(t)$ is zero-mean, complex, circular AWGN with power spectral density $\mathcal{N}_{0}$. An equivalent representation of the channel, central to our discussion, is in terms of the spreading function defined as

$$
H(\theta, \tau) \stackrel{\text { def }}{=} \int h(t, \tau) e^{-j 2 \pi \theta t} d t
$$

with the corresponding representation of $s(t)$ given by

$$
\begin{aligned}
s(t) & =\iint H(\theta, \tau)\left(\mathbf{F}_{\theta} \mathbf{T}_{\tau} x\right)(t) d \theta d \tau \\
& =\iint H(\theta, \tau) x(t-\tau) e^{j 2 \pi \theta t} d \theta d \tau .
\end{aligned}
$$

The spreading function $H(\theta, \tau)$ quantifies the time-frequency spreading produced by the channel- $\theta$ corresponds to the Doppler shifts introduced by the channel (temporal variations), and $\tau$ corresponds to the multipath delays.

The time-varying channel impulse response $h(t, \tau)$ is best modeled as a stochastic process, and the wide-sense stationary uncorrelated scatterer (WSSUS) model [1], [9] is widely used in which the temporal variations in $h(t, \tau)$ are represented as a stationary Gaussian process, and the channel responses at different lags (different scatterers) are uncorrelated (independent). The second-order statistics characterizing the channel are $^{1}$

$$
\begin{gathered}
\mathrm{E}\left\{H\left(\theta_{1}, \tau_{1}\right) H^{*}\left(\theta_{2}, \tau_{2}\right)\right\}=\Psi\left(\theta_{1}, \tau_{1}\right) \delta\left(\theta_{1}-\theta_{2}\right) \delta\left(\tau_{1}-\tau_{2}\right) \\
\Psi(\theta, \tau) \stackrel{\text { def }}{=} \mathrm{E}\left\{|H(\theta, \tau)|^{2}\right\}
\end{gathered}
$$

where $\delta(\cdot)$ denotes the Dirac delta function. The function $\Psi(\theta, \tau) \geq 0$ is called the scattering function and denotes the

\footnotetext{
${ }^{1}$ We assume a zero-mean channel (Rayleigh fading). Extension to Rician fading is straightforward.
} 
distribution of channel power as a function of multipath and Doppler shifts. The (essential) support of $\Psi(\theta, \tau)$ over $\tau$ is called the multipath spread of the channel, denoted by $T_{m}$. Similarly, the Doppler spread is the (one-sided) $\theta$-support of $\Psi(\theta, \tau)$, denoted by $B_{d}$.

Under our assumption of negligible intersymbol interference (ISI) $\left(T_{m} \ll T\right)$, the "one-shot detector" suffices which decodes each symbol independently; that is, the $p$ th symbol is decoded using $r(t), p T \leq t<(p+1) T$, only. Thus, without loss of generality, we base our discussion on the $p=0$ symbol

$$
\begin{aligned}
& r(t)=s(t)+n(t), \quad 0 \leq t<T \\
& s(t)=\int_{0}^{T_{m}} \int_{-B_{d}}^{B_{d}} \tilde{H}(\theta, \tau) q(t-\tau) e^{j 2 \pi \theta \tau} d \theta d \tau
\end{aligned}
$$

where

$$
\begin{aligned}
\tilde{H}(\theta, \tau) & =\int_{0}^{T} h(t, \tau) e^{-j 2 \pi \theta t} d t \\
& =T \int_{-B_{d}}^{B_{d}} H\left(\theta^{\prime}, \tau\right) e^{-j \pi\left(\theta-\theta^{\prime}\right) T} \operatorname{sinc}\left(\left(\theta-\theta^{\prime}\right) T\right) d \theta^{\prime}
\end{aligned}
$$

is the spreading function corresponding to $0 \leq t<T$, and $\operatorname{sinc}(x)=\sin (\pi x) / \pi x$.

\section{Fading and Diversity}

The degradation caused by the stochastic channel is best illustrated by the simple case of Rayleigh fading in which the channel exhibits no fluctuation over the signal duration and bandwidth; that is, $\tilde{H}(\theta, \tau)=\alpha \delta(\theta) \delta(\tau)$ in (13), where $\alpha$ is a zero-mean, Gaussian random variable with unit variance, and

$$
r(t)=\alpha q(t)+n(t) .
$$

Fig. 2 illustrates the degradation in performance due to fading for coherent antipodal signaling [1]. The BEP for the fading channel is depicted by the $L=1$ dashed curve in Fig. 2, whereas the solid curve corresponds to a nonfading AWGN channel. As evident, fading results in a loss of about $18 \mathrm{~dB}$ in SNR at BEP $=10^{-3}$.

Diversity techniques are employed in practice to combat the effects of fading [1]. The basic idea is to transmit the signal over multiple $(L>1)$ independent fading channels, while keeping the total power constant by transmitting at a lower power in each channel. Common diversity techniques include antenna diversity, time diversity, frequency diversity, and polarization diversity [1]-[3]. The dashed curves in Fig. 2 illustrate the performance improvement due to coherent diversity processing [1]. It is evident that performance improves monotonically with increasing $L$. In fact, as $L \rightarrow \infty$, the performance of coherent diversity reception converges to the performance over a nonfading AWGN channel [10]-[14].

\section{JOINT MULTIPATH-DOPPLER DIVERSITY}

The fast fading mobile wireless channel intrinsically affords diversity, and in this section we develop a CDMA framework that fully exploits it via joint multipath-Doppler processing.

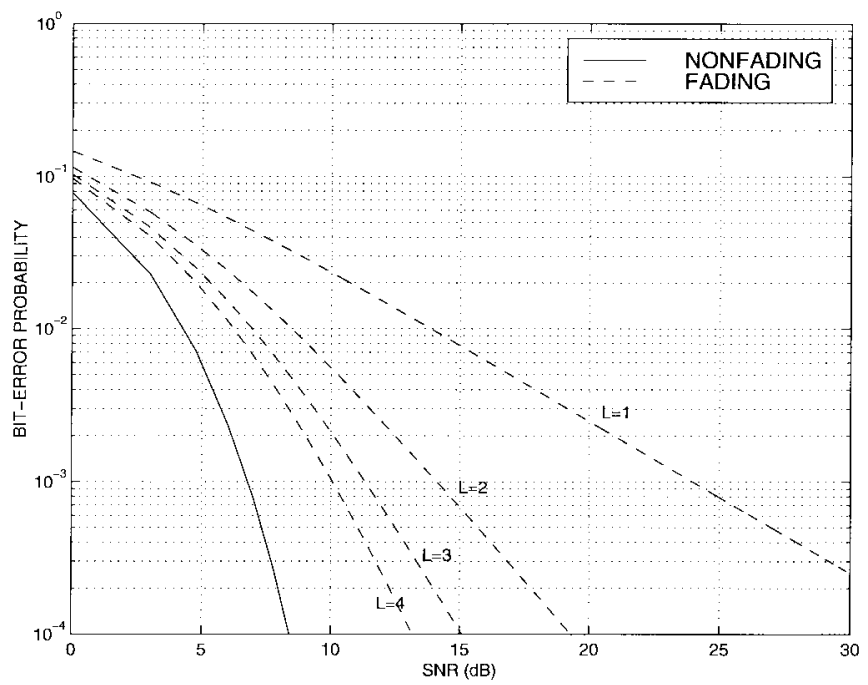

Fig. 2. BEP versus SNR for coherent antipodal signaling over AWGN (solid) and Rayleigh fading (dashed) channels. The dashed curves represent different levels $(L=1,2,3,4)$ of diversity. Monotonic improvement as a function of $L$ is evident.

Fundamental to our approach is a time-frequency decomposition of the channel into independent flat-fading channels. The channel decomposition naturally identifies an STFTbased generalization of the RAKE receiver for achieving joint multipath-Doppler diversity.

\section{A. Canonical Channel Decomposition}

As evident from (13), the received signal consists of a linear combination of time-shifted (multipath) and frequency-shifted (Doppler) copies of the transmitted signal. The time-frequency shifted copies of the transmitted signal produced by the channel provide an inherent diversity mechanism that can be exploited via appropriate signal processing. The following result describes a canonical representation of the channel and the received signal that plays a fundamental role in our framework for exploiting channel diversity. ${ }^{2}$

Theorem: The received signal $s(t)$ in (13) admits the finitedimensional representation

$$
s(t) \approx \frac{T_{c}}{T} \sum_{n=0}^{N} \sum_{k=-K}^{K} \hat{H}\left(\frac{k}{T}, n T_{c}\right) u_{k, n}(t)
$$

where $N=\left\lceil T_{m} / T_{c}\right\rceil \approx\left\lceil T_{m} B\right\rceil, K=\left\lceil B_{d} T\right\rceil$, and $\hat{H}(\theta, \tau)$ is a smoothed version of $H(\theta, \tau)$

$$
\begin{aligned}
\hat{H}(\theta, \tau)= & \frac{T}{T_{c}} \int_{0}^{T_{m}} \int_{-B_{d}}^{B_{d}} H\left(\theta^{\prime}, \tau^{\prime}\right) e^{-j \pi\left(\theta-\theta^{\prime}\right) T} \\
& \cdot \operatorname{sinc}\left(\left(\theta-\theta^{\prime}\right) T\right) \operatorname{sinc}\left(\left(\tau-\tau^{\prime}\right) / T_{c}\right) d \theta^{\prime} d \tau^{\prime} .
\end{aligned}
$$

The basis waveforms $u_{k, n}(t)$ 's are defined as

$$
u_{k, n}(t) \stackrel{\text { def }}{=}\left(\mathbf{F}_{k / T} \mathbf{T}_{n T_{c}} q\right)(t)=q\left(t-n T_{c}\right) e^{j(2 \pi k t / T)}
$$

${ }^{2}$ We note that similar sampled representations of the WSSUS channel have also been developed by Bello [9] and Kennedy [15] based on time and bandwidth constraints. However, our development and interpretation in the context of diversity signaling is quite different. 
and are approximately orthogonal to each other

$$
\left\langle u_{k, n}, u_{k^{\prime}, n^{\prime}}\right\rangle \approx T \delta_{k-k^{\prime}} \delta_{n-n^{\prime}}
$$

where $\delta_{k}$ denotes the Kronecker delta function.

Proof: See the Appendix.

Note that despite the smoothing in (17), the samples $\hat{H}(k / T, n T c)$ are uncorrelated for a sufficiently smooth $\Psi(\theta, \tau)$, as evident from

$$
\begin{aligned}
\mathrm{E}\{\hat{H} & \left.\left(\frac{k}{T}, n T_{c}\right) \hat{H}^{*}\left(\frac{l}{T}, m T_{c}\right)\right\} \\
= & \frac{T^{2}}{T_{c}^{2}} e^{-j \pi(k-l)} \iint \Psi(\theta, \tau) \operatorname{sinc}\left(\left(\frac{k}{T}-\theta\right) T\right) \\
& \cdot \operatorname{sinc}\left(\left(\frac{l}{T}-\theta\right) T\right) \operatorname{sinc}\left(\frac{n T_{c}-\tau}{T_{c}}\right) \\
& \cdot \operatorname{sinc}\left(\frac{m T_{c}-\tau}{T_{c}}\right) d \theta d \tau
\end{aligned}
$$

which directly follows from (17). By virtue of the orthogonality of the $u_{k, n}(t)$ 's, and the statistical independence of the channel coefficients $\hat{H}(\theta, \tau)$, the representation (16) effectively decomposes the channel into $(N+1) \times(2 K+1)$ independent, flat-fading (diversity) channels by appropriately sampling the multipath-Doppler plane. Fig. 3(a) illustrates the time-frequency sampling in (16). Note that the number of diversity channels is proportional to the product $T_{m} B_{d}\left(T / T_{c}\right)$. Thus, for fixed channel parameters $T_{m}$ and $B_{d}$, the level of diversity is proportional to the time-bandwidth product (TBP), $T B \approx\left(T / T_{c}\right)$, of the signaling waveform. This also illustrates the remarkable ability of CDMA systems to exploit channel diversity. For nonspread-spectrum signals, TBP $\approx$ 1 , since their bandwidth and duration cannot be controlled independently. However, for spread-spectrum signals, the TBP $\approx\left(T / T_{c}\right)=P$, the processing gain, which can be made arbitrarily large, in principle. The diversity gain is also a function of the power in the samples $\hat{H}\left(k / T, n T_{c}\right)$, as discussed later in Section IV-A. ${ }^{3}$

A few remarks are warranted about the loss of information in (16) due to the $(\theta, \tau)$-sampling. Due to the time- and bandlimited nature of $q(t)$, the receiver only "sees" a corresponding time- and band-limited version of the channel, which in turn justifies the sampling of the spreading function in (16). In fact, the approximation in (16) can be made arbitrarily close by oversampling in multipath and by increasing the number of terms in the summation. However, virtually all the signal energy is captured by $N=\left\lceil T_{m} / T_{c}\right\rceil$ multipath and $K=\left\lceil T B_{d}\right\rceil$ Doppler components. Furthermore, (16) is a Karhunen-Loève-like expansion of the received signal $s(t)$ : the $\hat{H}\left(k / T, n T_{c}\right)$ 's are uncorrelated random variables, and the $u_{k, n}(t)$ 's are (roughly) orthogonal waveforms. The approximate orthogonality of the $u_{k, n}(t)$ 's is illustrated in Fig. 3(b) which shows a three-dimensional plot of the timefrequency correlation function

$$
\operatorname{STFT}_{q}^{(q)}\left(\frac{k}{T}, n T_{c}\right)
$$

\footnotetext{
${ }^{3}$ From the perspective of received signal power, (16) should be interpreted as follows: the total received power remains constant-it is distributed in the $(N+1) \times(2 K+1)$ channel samples according to $\Psi(\theta, \tau)$.
}

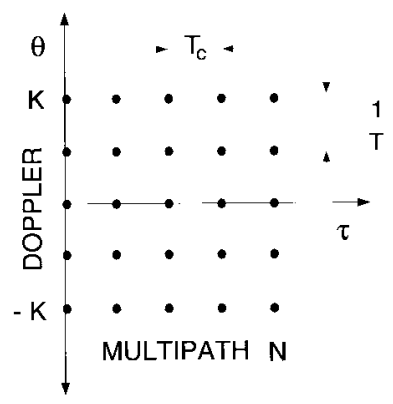

(a)

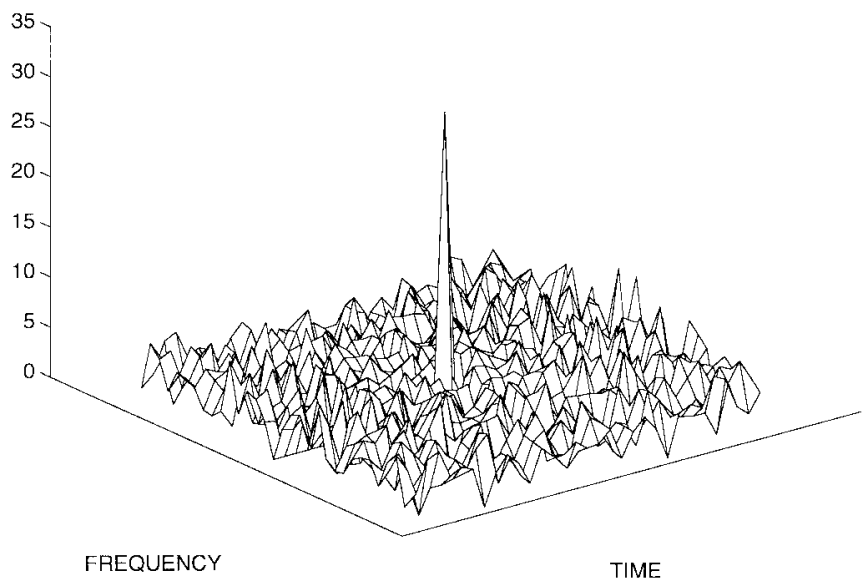

(b)

Fig. 3. (a) Sampling of the time-frequency plane to create multipath-Doppler diversity channels. (b) Sampled time-frequency correlation function of a spread-spectrum signaling waveform (length-31 M-sequence).

$$
=\left\langle q, \mathbf{F}_{k / T} \mathbf{T}_{n T_{c}} q\right\rangle=\int q(t) q^{*}\left(t-n T_{c}\right) e^{-j(2 \pi k t / T)} d t
$$

of a spread-spectrum waveform generated from a length-31 M-sequence [1].

\section{B. Optimal Time-Frequency Receiver Structures}

The canonical signal representation (16) clearly identifies the correlator waveforms needed to process the independent multipath-Doppler components

$$
\begin{aligned}
u_{k, n}^{m}(t) \stackrel{\text { def }}{=}\left(\mathbf{F}_{k / T} \mathbf{T}_{n T_{c}} q^{m}\right)(t)=q^{m}\left(t-n T_{c}\right) e^{j(2 \pi k t / T)}, & \\
k & =-K,-K+1, \cdots, K, \\
n & =0,1, \cdots, N .
\end{aligned}
$$

Recalling the definition of the STFT in (2), we note that the correlator outputs can be computed via sampled STFT's; that is, the sufficient statistics are given by

$$
Z_{k, n}^{m} \stackrel{\text { def }}{=}\left\langle r, u_{k, n}^{m}\right\rangle=\mathbf{S T F T}_{r}^{\left(q^{m}\right)}\left(\frac{k}{T}, n T_{c}\right) .
$$

The sufficient statistics $Z_{k, n}^{m}$ may be combined coherently if the channel coefficients $\hat{H}\left(k / T, n T_{c}\right)$ are available at the receiver. ${ }^{4}$ The optimal test statistic, which is applicable to both

\footnotetext{
${ }^{4}$ Pilot-based estimates of $\hat{H}\left(k / T, n T_{c}\right)$ may be available-more discussion in the next subsection.
} 


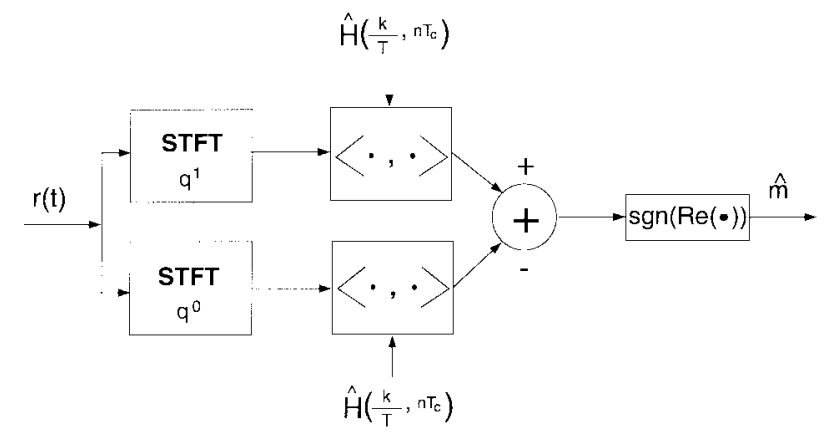

(a)

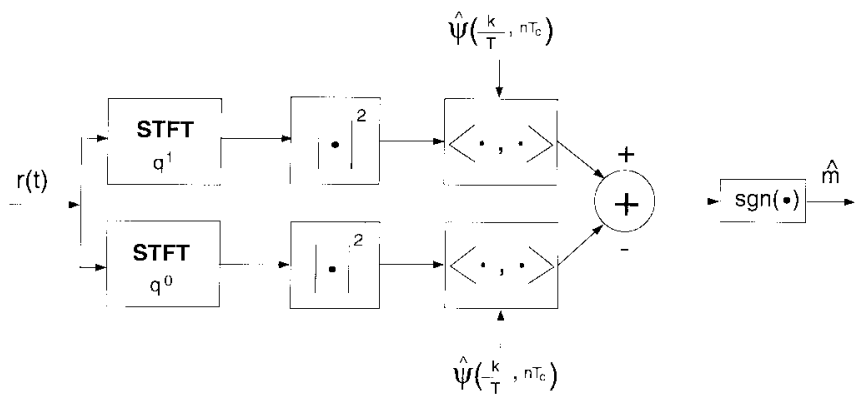

(b)

Fig. 4. STFT-based detector structures (time-frequency RAKE receivers) for exploiting joint multipath-Doppler diversity: (a) coherent processing and (b) noncoherent processing.

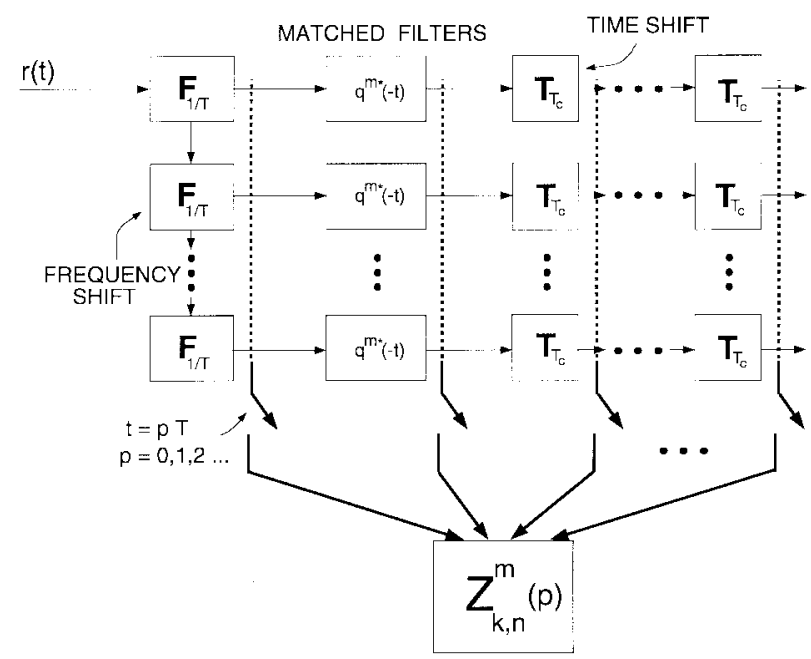

Fig. 5. Implementation of sampled STFT using a bank of identical matched filters determined by the signaling waveforms. $Z_{k, n}^{m}(p)$ denotes the sufficient statistics for the $p$ th symbol; that is, $Z_{k, n}^{m}(p)=\left\langle r_{p}, u_{k, n}^{m}\right\rangle$, where $r_{p}(t)=r(t+p T)$. Note that each matched filter branch corresponds to a conventional RAKE receiver.

antipodal or orthogonal signaling, is given by

$$
\begin{gathered}
Z_{C}=\operatorname{sgn}\left(\operatorname { r e a l } \left\{\sum_{n=0}^{N} \sum_{k=-K}^{K} \hat{H}^{*}\left(\frac{k}{T}, n T_{c}\right)\right.\right. \\
\left.\left.\cdot\left[Z_{k, n}^{1}-Z_{k, n}^{0}\right]\right\}\right) .
\end{gathered}
$$

Fig. 4(a) illustrates the STFT-based detector structure for coherent processing.
If channel coefficients are not available at the receiver, but only the power in the channel coefficients is known, noncoherent processing may be used. The optimal noncoherent (quadratic) test statistic is given by

$$
\begin{gathered}
Z_{Q}=\operatorname{sgn}\left(\sum_{n=0}^{N} \sum_{k=-K}^{K} \hat{\Psi}\left(\frac{k}{T}, n T_{c}\right)\right. \\
\left.\cdot\left[\left|Z_{k, n}^{1}\right|^{2}-\left|Z_{k, n}^{0}\right|^{2}\right]\right) \\
\hat{\Psi}(\theta, \tau) \stackrel{\text { def }}{=} \frac{\|q\|^{2} \mathrm{E}\left\{\left.\hat{H}(\theta, \tau)\right|^{2}\right\}}{\|q\|^{2} \mathrm{E}\left\{\left.\hat{H}(\theta, \tau)\right|^{2}\right\}+\mathcal{N}_{0}} .
\end{gathered}
$$

If the channel power profile is not known exactly, equalgain noncoherent combining may be employed, which assumes uniform power in the different multipath-Doppler components $(\hat{\Psi}(\theta, \tau)=1)$. Fig. 4(b) illustrates the STFT-based noncoherent detector structure which, of course, is only valid with orthogonal signaling.

1) Remarks on Optimality: Both the coherent $\left(Z_{C}\right)$ and noncoherent $\left(Z_{Q}\right)$ detectors are optimal in the sense of minimum BEP. However, they assume different amount of information about the channel. $Z_{C}$ requires knowledge of the actual channel coefficients, whereas $Z_{Q}$ only requires knowledge of the second-order channel statistics. With exact knowledge of the channel coefficients, $Z_{C}$ would obviously perform better than $Z_{Q}$. However, in practice the channel has to be estimated, such as through a pilot transmission. If the channel coefficients are estimated from the received waveform $r(t)$, it can be shown that the corresponding $Z_{C}$ cannot outperform $Z_{Q}$. This is related to the estimator-correlator interpretation of $Z_{Q}{ }^{5}$ However, if the channel is estimated from a pilot transmission at a higher SNR compared to $r(t)$, then $Z_{C}$ can perform better than $Z_{Q}$. In general, the performance of a pilot-based coherent receiver would lie somewhere between that of $Z_{C}$ (with perfect channel estimates) and $Z_{Q}$.

2) Special Cases of the Time-Frequency Receivers: There are two important special cases of the proposed time-frequency receivers. One is the well-known RAKE receiver that exploits multipath diversity, and the other is a new receiver structure for exploiting Doppler diversity, which we refer to as the "Doppler RAKE receiver."

The RAKE receiver corresponds to the samples on the multipath axis in Fig. 3(a); that is, it achieves $(N+1)$-point multipath diversity as opposed to the $(N+1) \times(2 K+1)$-point diversity of the time-frequency RAKE receiver. The decision statistics for the RAKE receiver are given by (24) and (25) by setting $K=0$. The RAKE receiver is optimal for slowfading $\left(T B_{d} \approx 0\right)$, frequency-selective $\left(T_{m} \geq T_{c}\right)$ scenarios and is implicitly based on a time-invariant channel model: $H(\theta, \tau)=\delta(\theta) g(\tau)$. In fact, practical implementations of the RAKE receiver rely on slow fading to obtain accurate channel estimates by averaging over several symbols [1], [5]. Indeed, the errors incurred in channel estimation are primarily

\footnotetext{
${ }^{5} Z_{Q}$ inherently forms a minimum-mean-squared-error estimate of the channel coefficients based on $r(t)$, which is also optimal from a detection viewpoint.
} 
responsible for the performance degradation of the RAKE receiver under fast fading [3]-[6].

The Doppler RAKE receiver is the dual of the conventional RAKE receiver in that it samples the Doppler axis to achieve $(2 K+1)$-point diversity [see Fig. 3(a)]. It is optimal for fastfading $^{6}\left(T B_{d} \geq 0.01\right)$, frequency-nonselective $\left(T_{m} \ll T_{c}\right)$ scenarios and assumes a frequency-invariant channel model: $H(\theta, \tau)=M(\theta) \delta(\tau)$. The Doppler RAKE decision statistics are given by (24) and (25) by setting $N=0$.

3) Implementational Complexity: Fig. 5 illustrates the implementation of the sampled STFT via a bank of identical matched filters determined by the signaling waveforms. The received signal $r(t)$, modulated to different Doppler frequencies, is fed to the different matched filters. Note that each matched filter admits a tapped delay-line implementation of the RAKE receiver. Thus, time-frequency RAKE receivers can be efficiently implemented via a bank of conventional RAKE receivers - their computational complexity is $(2 K+1)$ times that of the conventional RAKE receiver.

\section{Discussion}

The key idea behind our time-frequency-based framework for fast fading channels is to exploit the cause of degradation in existing systems-Doppler shifts-to provide additional diversity, in conjunction with multipath diversity. In this section, we demonstrate the practical viability and potential performance gains of joint multipath-Doppler diversity and identify several system modalities that can benefit from it.

\section{A. Potential Performance Gains}

We demonstrate in this section that the relatively small Doppler spreads encountered in practice can be leveraged into substantial diversity gains via appropriate signal processing. In practice, performance degradation occurs in existing systems for values of $T B_{d}$ as low as 0.01 [4], [5]. Such values of $T B_{d}$ are commonly encountered. For example, $T B_{d} \approx 0.013$ at a data rate of $10 \mathrm{kHz}$, carrier frequency of $1.8 \mathrm{GHz}$, and maximum mobile speed of $50 \mathrm{~m} / \mathrm{h}$. More importantly, at modestly larger values of $T B_{d}\left(T B_{d} \geq 0.2\right)$, significant gains $(\geq 3 \mathrm{~dB}$ in SNR) can be achieved by exploiting Doppler diversity. As elaborated in Section IV-B, there are several system modalities in which such gains can be achieved by attaining desired values of $T B_{d}$ via appropriate signaling and reception.

Such remarkable diversity gains at relatively small Doppler spreads are due to the fact that a large fraction of the maximum gain due to additional diversity components is attained at relatively small fractions of the total power in those components. For illustration, consider the practically relevant situation in which $0<T B_{d}<1$, resulting in $K=1$ in (16). Using (16) and (17), it can be readily shown that the power in the signal component of the time-frequency correlator outputs (23) is given by

$$
\begin{gathered}
\mathrm{E}\left\{\left|\left\langle s, u_{k, n}\right\rangle\right|^{2}\right\} \approx \\
\|q\|^{4} \int_{0}^{T_{m}} \int_{-B_{d}}^{B_{d}} \Psi(\theta, \tau) \operatorname{sinc}^{2}\left(\tau / T_{c}-n\right) \\
\cdot \operatorname{sinc}^{2}(\theta T-k) d \theta d \tau .
\end{gathered}
$$

\footnotetext{
${ }^{6}$ See Section IV-A.
}

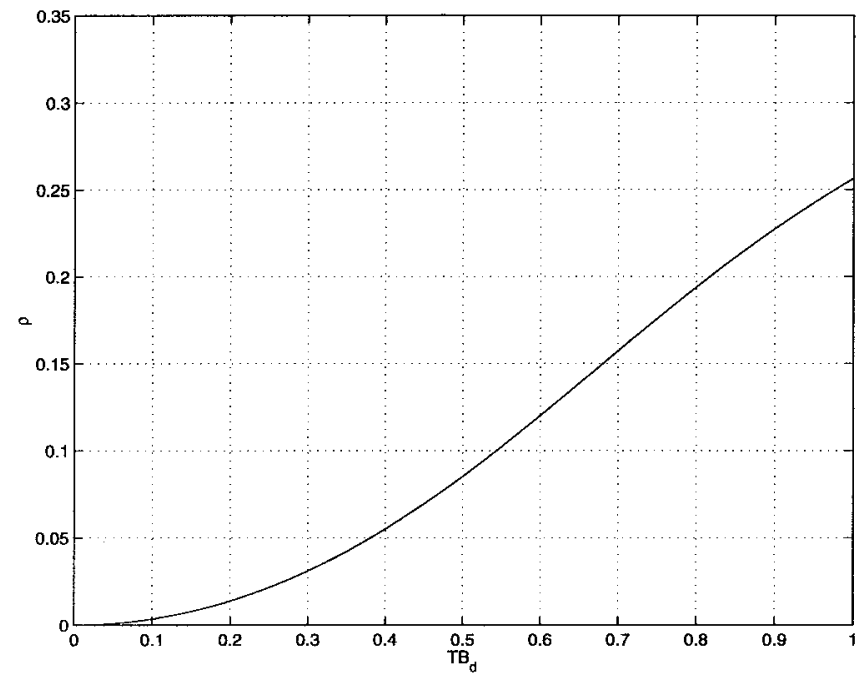

Fig. 6. The variation in $\rho$ as a function of the product $T B_{d}$.

For simplicity, assume uniform channel power distribution as a function of Doppler variable: $\Psi(\theta, \tau)=\Psi(\tau)$ for $(\theta, \tau) \in\left(-B_{d}, B_{d}\right) \times\left[0, T_{m}\right) .^{7}$ For each fixed multipath index $n$, the ratio of the power in the $k= \pm 1$ Doppler components to the total power in the three $(k=0, \pm 1)$ components is

$$
\begin{aligned}
\rho\left(T B_{d}\right) & \stackrel{\text { def }}{=} \frac{\mathrm{E}\left\{\left|\left\langle s, q_{ \pm 1, n}\right\rangle\right|^{2}\right\}}{\mathrm{E}\left\{\left|\left\langle s, q_{0, n}\right\rangle\right|^{2}\right\}+2 \mathrm{E}\left\{\left|\left\langle s, q_{ \pm 1, n}\right\rangle\right|^{2}\right\}} \\
& =\frac{\int_{-T B_{d}}^{T B_{d}} \operatorname{sinc}^{2}(\beta-1) d \beta}{\int_{-T B_{d}}^{T B_{d}} \operatorname{sinc}^{2}(\beta) d \beta+2 \int_{-T B_{d}}^{T B_{d}} \operatorname{sinc}^{2}(\beta-1) d \beta}
\end{aligned}
$$

Note that $0 \leq \rho\left(T B_{d}\right) \leq 1 / 3$ and increases monotonically with $T B_{d}$ as shown in Fig. 6. $\rho=0$ at $T B_{d}=0$, corresponding to $(N+1)$ equal-power multipath components. Whereas, $\rho \approx 1 / 4$ at $T B_{d}=1$, corresponding to $3(N+1)$ multipathDoppler diversity components with a $(1 / 4,1 / 2,1 / 4)$ power distribution as a function of the Doppler index $k$. The gain due to joint multipath-Doppler diversity increases as $\rho \rightarrow 1 / 4$ $\left(T B_{d} \rightarrow 1\right)$.

Fig. 7 illustrates the performance gains due to joint multipath-Doppler diversity as a function of $\rho$ for two $(N=1)$ multipath components and coherent processing. ${ }^{8}$ As evident, an SNR gain of $8.5 \mathrm{~dB}\left(\mathrm{BEP}=10^{-4}\right)$ is achievable $(\rho=1 / 4)$ with the time-frequency RAKE receiver as compared to the RAKE receiver. However, the remarkable fact is that more than a third $(3 \mathrm{~dB})$ of the $8.5 \mathrm{~dB}$ gain is achieved at about $1.25 \%$ of the total power in the $k= \pm 1$ Doppler components $(\rho=0.0125)$. Similarly, more than $5 \mathrm{~dB}$ SNR gain is achieved at about $5 \%$ of the total power in the Doppler components $(\rho=0.05)$. As evident from Fig. 6 , the values of $\rho=0.0125,0.05$, and 0.1 are achieved at relatively small

\footnotetext{
${ }^{7}$ We note that the total signal power in the correlator outputs remains constant for a given SNR-the distribution of power as a function of $k$ (Doppler) depends on $T B_{d}$ as in (28).

${ }^{8} \mathrm{BEP}$ calculations are based on the analysis of RAKE receiver in $[1, \mathrm{pp}$. 798-806].
} 


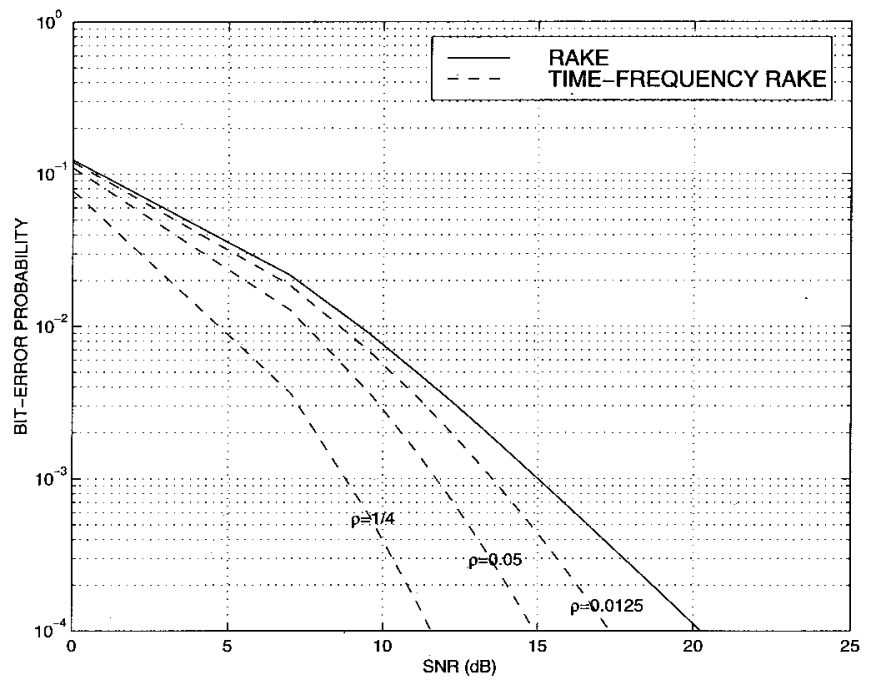

Fig. 7. Joint multipath-Doppler diversity gains as a function of $\rho$ for coherent antipodal signaling (perfect channel knowledge) and $N=2$ multipath components. The performance of the RAKE receiver is under (ideal) slow fading $\left(T B_{d}=0, \rho=0\right)$.

values of $T B_{d}: \approx 0.2,0.39$, and 0.6 , respectively. We note that similar gains, implicitly due to Doppler diversity, have been reported in other non-CDMA systems as well [16]. ${ }^{9}$ Similar instance of small causes leading to large effects have been observed in other related contexts as well [18], [19].

Finally, Fig. 8 illustrates the multipath-Doppler diversity gains realistically achievable in practice compared to the RAKE receiver with two multipath components. Note that the performance of the time-frequency RAKE receiver is based on noncoherent processing which provides a lower bound on the achievable gains. The coherent RAKE receiver signifies the optimal performance of existing systems under slow fading, and the noncoherent RAKE receiver typifies the degraded performance of existing systems under fast fading due to errors in channel estimation. As evident, the time-frequency RAKE receiver can provide substantial gains even with noncoherent processing (to avoid channel estimation). Gains of 3.5-6.5 dB in SNR $\left(\mathrm{BEP}=10^{-4}\right)$ are achievable for $0.025<\rho<0.1$ $\left(0.27<T B_{d}<0.55\right)$. As discussed in the next section, such values of $T B_{d}$ are attainable in many practical scenarios by appropriate signaling and/or reception.

It is worth noting that the above analysis assumes a uniform Doppler power spectrum. A more realistic "bathtub-shaped" Doppler profile [20] would yield comparable gains at even smaller values of $T B_{d}$. Furthermore, for $1<T B_{d}<2$, fivefold $(K=2)$ Doppler diversity (as opposed to three-fold with $\left.0<T B_{d}<1\right)$ can be attained. In addition, by explicitly accounting for fast fading, multipath-Doppler processing does not suffer from BEP floors and promises even bigger SNR gains at lower BEP's.

\section{B. Applicable Scenarios}

As noted in the previous section, values of $T B_{d}$ in the range 0.01-0.015 are commonly encountered in mobile scenarios

\footnotetext{
${ }^{9}$ However, we note that due to the relatively small $(\ll 1)$ values of $T_{m} B_{d}$ in practice [17], nonspread-spectrum systems cannot exploit both multipath and Doppler diversity simultaneously.
}

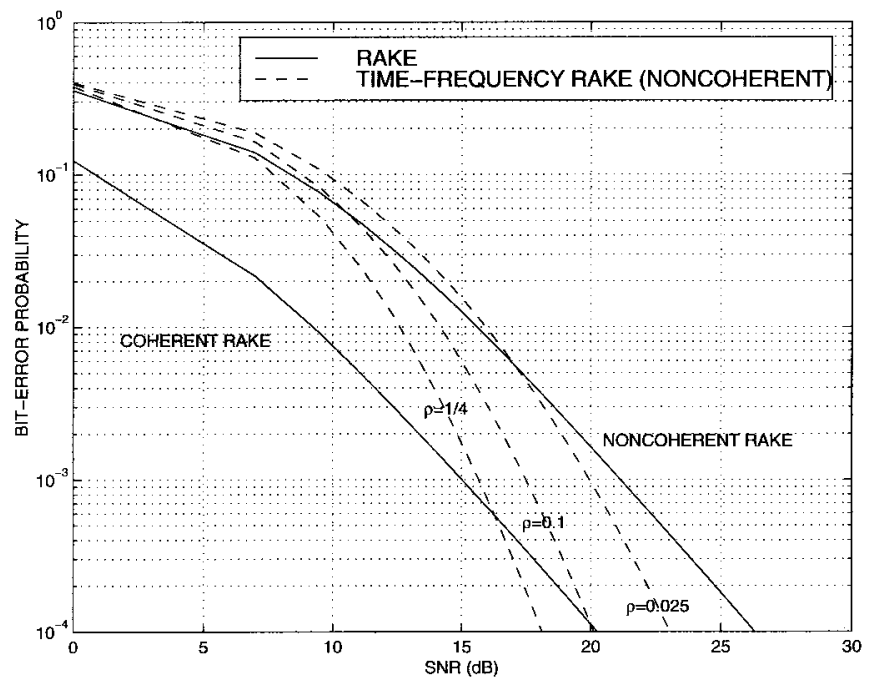

Fig. 8. Joint multipath-Doppler diversity gains as a function of $\rho$ for noncoherent equal-gain combining (no channel knowledge) and $N=2$ multipath components. The coherent RAKE receiver (antipodal signaling) signifies the optimal performance of existing systems under slow fading. The noncoherent RAKE receiver (orthogonal signaling) typifies the degraded performance of existing systems under fast fading. Note that the noncoherent time-frequency RAKE receiver provides a lower bound on the performance of proposed systems.

and can significantly degrade the performance of existing systems. However, fast fading can be leveraged into substantial multipath-Doppler diversity gains at moderately larger values of $T B_{d}(0.2-0.6)$. In this section, we discuss system modalities in which desired values of $T B_{d}$ can be achieved by making $T$ effectively longer.

A direct way to attain larger values of $T B_{d}$ is to employ time-selective signaling by increasing the duration $T$ [12], [13], [21]. In order to keep the data rate at a prescribed value, successive symbols may have to overlap in time, resulting in ISI. However, initial results show that the excellent correlation properties of pseudorandom sequences make the ISI virtually negligible [12], [13]. Moreover, simulations based on Jakes model [20] (reported in [12] and [13]) show that this approach can indeed deliver the diversity gains predicted by the analysis in the previous section. For example, a 3.5-dB SNR gain at $\mathrm{BEP}=10^{-4}$ can be achieved by using symbols that are 16 times longer than the conventional symbol (to attain $T B_{d} \approx$ 0.2 ). For a detailed discussion, we refer the reader to [13]. ${ }^{10}$ We note that this time-selective signaling approach is similar in spirit, and provides an alternative, to the precoding/spreadsignature ideas proposed in [11] and [22] (details in [13]).

In many practical systems, bits are processed in frames for the purpose of interleaving. For example, in the interim standard IS-95, processing is done in frames of 192 bits which corresponds to a frame duration of about $T_{f}=20 \mathrm{~ms}$ [2]. This yields $1<T_{f} B_{d}<2$ for a typical $B_{d}$ range of $60-100$ $\mathrm{Hz}$, which strongly suggests block-based processing to make the channel time-selective for exploiting Doppler diversity ( $K=2$ or $K=3$ ) - jointly decoding the bits in a fraction of the frame via sequence decoding to make the effective

\footnotetext{
${ }^{10}$ Note that the delay introduced by time-selective signaling is inconsequential compared to delays due to other system requirements, such as interleaving.
} 
$T$ longer. Given the observation that significant gains due to Doppler diversity may be achieved for relatively small values of $T B_{d}$, relatively small block sizes may be adequate in such an approach. Moreover, the fact that the channel remains virtually constant over several symbols may also be used to develop efficient sequence decoding algorithms. Such techniques for exploiting joint multipath-Doppler diversity are currently under investigation.

Clearly, transmission at relatively low data rates facilitates the exploitation of Doppler diversity. Thus, the techniques developed in this paper may also be used in underwater acoustic systems [23]-[25] and emerging modalities such as multicarrier CDMA [26]. ${ }^{11}$

\section{CONCLUSIONS}

Signal fading produced by the wireless channel is one of most significant factors limiting the performance of mobile communication systems. The mobile wireless channel inherently affords diversity that can be exploited via the use of large-TBP signaling waveforms to combat fading. In this context, spread-spectrum CDMA systems have a distinct advantage over other systems, such as time-division or frequency-division multiple-access systems. However, existing CDMA systems based on the RAKE receiver only partly exploit channel diversity: they are optimal for slow fading scenarios and achieve multipath diversity. Consequently, existing systems perform poorly under fast fading and exhibit BEP floors due to mismatch with channel characteristics.

The new CDMA framework proposed in this paper fully exploits the inherent channel diversity via joint time-frequency processing. Unlike existing receivers that treat Doppler as a nuisance, our framework demonstrates that Doppler is in fact another dimension for diversity that should be exploited to combat fading. The proposed time-frequency-based system is matched to the fast-fading channel characteristics and attains a larger TBP to exploit joint multipath-Doppler diversity in an optimal fashion. Performance analysis indicates that even the relatively modest Doppler spreads encountered in practice can be leveraged into substantial gains using our approach. Typically, joint multipath-Doppler processing doubles or triples the level of diversity compared to existing systems, resulting in significant improvements in SNR. Moreover, the proposed systems do not suffer from BEP floors under fast fading and promise even larger SNR gains at lower BEP's that may be required for certain transmission formats, such as data.

Due to the central nature of the channel, joint multipathDoppler processing can be integrated into many aspects of system design and promises improved performance due to the inherently higher level of diversity. In particular, the basic framework can be extended to develop techniques for synchronization and channel estimation [21], [27] and multiuser detection [28], [29]. Moreover, our approach can also be used to develop algorithms for tracking channel statistics, providing an alternative to Kalman filter-based algorithms proposed in

\footnotetext{
${ }^{11}$ The different "Doppler channels" [see Fig. 3(a)] should not be confused with the different channels in a multicarrier system. Our approach used in conjunction with a multicarrier system would correspond to Doppler channels associated with each subcarrier.
}

[23], [24], [30], and [31]. Finally, powerful coding techniques developed for the AWGN channel may also be integrated into our approach since the high level of diversity makes the fading channel effectively look like an AWGN channel [12], [13].

\section{APPENDIX}

\section{PROOF OF THE THEOREM}

We first derive the signal representation (16). Recall from (13) that

$$
s(t)=\int_{0}^{T_{m}} \tilde{h}(t, \tau) q(t-\tau) d \tau, \quad 0 \leq t<T
$$

where $\tilde{h}(t, \tau)=h(t, \tau) I_{[0, T)}(t)$, which admits the Fourier series

$$
\begin{gathered}
\tilde{h}(t, \tau) \\
=\sum_{k} c_{k}(\tau) e^{j(2 \pi k t / T)} \approx \frac{1}{T} \sum_{k=-K}^{K} \tilde{H}\left(\frac{k}{T}, \tau\right) e^{j(2 \pi k \cdot t / T)} \\
c_{k}(\tau) \stackrel{\text { def }}{=} \frac{1}{T} \int_{0}^{T} h(t, \tau) e^{-j(2 \pi k t / T)} d t=\frac{1}{T} \tilde{H}\left(\frac{k}{T}, \tau\right)
\end{gathered}
$$

with $\tilde{H}(\theta, \tau)$ defined in (14). Since the one-sided width of the mainlobe of $\operatorname{sinc}(\theta T)$ is $1 / T$, most of the energy in (30) is contained in $|k| \leq K, K=\left\lfloor\left(B_{d}+1 / T\right) T\right\rfloor=\left\lceil B_{d} T\right\rceil$ components. Substituting (30) in (29) we get

$$
\begin{aligned}
s(t) & \approx \frac{1}{T} \sum_{k=-K}^{K} e^{j(2 \pi k t / T)} \int_{0}^{T_{m}} \tilde{H}\left(\frac{k}{T}, \tau\right) q(t-\tau) d \tau \\
& =\frac{1}{T} \sum_{k=-K}^{K} e^{j(2 \pi k t / T)} \int \tilde{G}\left(\frac{k}{T}, \nu\right) Q(\nu) e^{j 2 \pi \nu t} d \nu
\end{aligned}
$$

where

$$
\tilde{G}(\theta, \nu)=\int \tilde{H}(\theta, \tau) e^{-j 2 \pi \nu \tau} d \tau
$$

and

$$
Q(f)=\int q(t) e^{-j 2 \pi f t} d t .
$$

Since $Q(f)$ is approximately bandlimited to $|f| \leq W \approx$ $1 / 2 T_{c}$, only the approximation

$$
\hat{G}(\theta, \nu)=\tilde{G}(\theta, \nu) I_{\left(-1 / 2 T_{c}, 1 / 2 T_{c}\right)}(\nu)
$$

of $\tilde{G}(\theta, \tau)$ matters in (32), which admits a Fourier series

$$
\begin{aligned}
\hat{G}(\theta, \nu) & =\sum_{n} c_{n}(\theta) e^{-j 2 \pi n T_{c} \nu} \\
& \approx T_{c} \sum_{n=0}^{N} \hat{H}\left(\theta, n T_{c}\right) e^{-j 2 \pi n T_{c} \nu} \\
c_{n}(\theta) & \stackrel{\text { def }}{=} T_{c} \int \hat{G}(\theta, \nu) e^{j 2 \pi n T_{c} \nu} d \nu=T_{c} \hat{H}\left(\theta, n T_{c}\right)
\end{aligned}
$$


where

$$
\begin{aligned}
\hat{H}(\theta, \tau)= & \int_{-1 / 2 T_{c}}^{1 / 2 T_{c}} \tilde{G}(\theta, \nu) e^{j 2 \pi \nu \tau} d \nu \\
= & \frac{1}{T_{c}} \int_{0}^{T_{m}} \tilde{H}\left(\theta, \tau^{\prime}\right) \operatorname{sinc}\left(\left(\tau-\tau^{\prime}\right) / T_{c}\right) d \tau^{\prime} \\
= & \frac{T}{T_{c}} \int_{0}^{T_{m}} \int_{-B_{d}}^{B_{d}} H\left(\theta^{\prime}, \tau^{\prime}\right) e^{-j \pi\left(\theta-\theta^{\prime}\right) T} \\
& \cdot \operatorname{sinc}\left(\left(\theta-\theta^{\prime}\right) T\right) \operatorname{sinc}\left(\left(\tau-\tau^{\prime}\right) / T_{c}\right) d \theta^{\prime} d \tau^{\prime} .
\end{aligned}
$$

Thus, most of the energy in (35) is contained in $N=$ $\left\lfloor\left(T_{m}+T_{c}\right) / T_{c}\right\rfloor=\left\lceil T_{m} / T_{c}\right\rceil$ terms. Substituting (35) in (32) we get (16).

We now demonstrate the approximate orthogonality of the $u_{k, n}(t)$ 's. First, we note from (18) that up to a phase factor, $\left\langle u_{k, n}, u_{k^{\prime}, n^{\prime}}\right\rangle=\left\langle u_{0,0}, u_{k-k^{\prime}, n-n^{\prime}}\right\rangle$. Thus, it is sufficient to consider the time-frequency correlation function

$$
\begin{aligned}
\left\langle q, \mathbf{F}_{\theta} \mathbf{T}_{\tau} q\right\rangle= & \sum_{l=0}^{P-1} \sum_{m=0}^{P-1} c[l] c[m] \int v\left(t-l T_{c}\right) \\
& \cdot v\left(t-\tau-m T_{c}\right) e^{-j 2 \pi \theta t} d t
\end{aligned}
$$

where we have used the expression in (6) for $q(t)$. Evaluating the time-frequency correlation at $\theta=k / T$ and $\tau=n T_{c}$, we get

$$
\begin{aligned}
\langle q, & \left.\mathbf{F}_{k / T} \mathbf{T}_{n T_{c}} q\right\rangle \\
= & \sum_{l=0}^{P-1} \sum_{m=0}^{P-1} c[l] c[m] \int v\left(t-l T_{c}\right) \\
& \cdot v\left(t-(m+n) T_{c}\right) e^{-j(2 \pi k t / T)} d t \\
= & \sum_{l=0}^{P-1} c[l] c[l-n] \int v^{2}\left(t-l T_{c}\right) e^{-j(2 \pi k t / T)} d t \\
= & \sum_{l=0}^{P-1} c[l] c[l-n] \int_{l T_{c}}^{(l+1) T_{c}} e^{-j(2 \pi k t / T)} d t \\
= & T_{c} e^{-j\left(\pi k T_{c} / T\right)} \operatorname{sinc}\left(\frac{\pi k T_{c}}{T}\right) \\
& \cdot \sum_{l=0}^{P-1} c[l] c[l-n] e^{-j\left(2 \pi k l T_{c} / T\right)} .
\end{aligned}
$$

Thus,

$$
\begin{aligned}
\left|\left\langle q, \mathbf{F}_{k / T} \mathbf{T}_{n T_{c}} q\right\rangle\right| & \leq T_{c}\left|\sum_{l=0}^{P-1} c[l] c[l-n] e^{-j\left(2 \pi k l T_{c} / T\right)}\right| \\
& =T_{c}\left|\sum_{l=0}^{P-1} c[l] c[l-n] e^{-j(2 \pi k l / P)}\right|
\end{aligned}
$$

For $n=0$, the right-hand side is equal to $T_{c} \mid \sum_{l=0}^{P-1}$ $e^{-j(2 \pi k l / P)} \mid=T_{c} P \delta_{k}=T \delta_{k}$. For $n \neq 0$, the variation in the exponential in (40) is much slower than the variation in the spreading codes for small values of $k$, which is typically the case since $K$ is typically much smaller than $P$. Hence, we have

$$
\left|\left\langle q, \mathbf{F}_{k / T} \mathbf{T}_{n T_{c}} q\right\rangle\right| \approx T_{c}\left|\sum_{l=0}^{P-1} c[l] c[l-n]\right| \approx 0
$$

which shows that for $n \neq 0$, the orthogonality of the $u_{k, n}(t)$ 's follows from the temporal orthogonality properties of the spreading code, which improve with increasing length of the code.

\section{REFERENCES}

[1] J. G. Proakis, Digital Communications, 3rd ed. New York: McGrawHill, 1995.

[2] T. S. Rappaport, Wireless Communications. Englewood Cliffs, NJ: Prentice Hall, 1996.

[3] B. Sklar, "Rayleigh fading channels in mobile digital communication system-Part II: Mitigation," IEEE Commun. Mag., pp. 103-109, July 1997.

[4] H. Andoh, M. Sawahashi, and F. Adachi, "Channel estimation using time multiplexed pilot symbols for coherent Rake combining for DS-CDMA mobile radio," in Proc. PIMRC'97, pp. 954-958.

[5] J. K. Cavers, "An analysis of pilot symbol assisted modulation for Rayleigh fading channels," IEEE Trans. Veh. Technol., vol. VT-40, pp. 689-693, Nov. 1991.

[6] F. Ling, "Coherent detection with reference-symbol based estimation for direct sequence CDMA uplink communication," in Proc. VTC'93, pp. $400-403$.

[7] F. Hlawatsch and G. F. Boudreaux-Bartels, "Linear and quadratic timefrequency signal representations," IEEE Signal Processing Mag., Apr. 1992.

[8] L. Cohen, Time-Frequency Analysis. Englewood Cliffs, NJ: Prentice Hall, 1995.

[9] P. A. Bello, "Characterization of randomly time-variant linear channels," IEEE Trans. Commun. Syst., vol. CS-11, pp. 360-393, 1963.

[10] J. Ventura-Traveset, G. Caire, E. Biglieri, and G. Taricco, "Impact of diversity reception on fading channels with coded modulation-Part I: Coherent detection," IEEE Trans. Commun., vol. 45, pp. 563-572, May 1997.

[11] G. W. Wornell, "Spread-response precoding for communication over fading channels," IEEE Trans. Inform. Theory, vol. 42, pp. 488-501, Mar. 1996

[12] S. Bhasyam, A. M. Sayeed, and B. Aazhang, "Time-selective signaling and reception for multipath fading channels," presented at 1998 IEEE Int. Symp. Information Theory.

[13] _ "Time-selective signaling and reception for communication over multipath fading channels," IEEE Trans. Commun., to be published.

[14] A. J. Viterbi, CDMA: Principles of Spread Spectrum Communications. Reading, MA: Addison-Wesley, 1995.

[15] R. Kennedy, Fading Dispersive Communication Channels. New York: Wiley, 1969.

[16] B. Hart and D. P. Taylor, "Extended MLSE diversity receiver for the time- and frequency-selective channel," IEEE Trans. Commun., vol. 45, pp. 322-333, Mar. 1997

[17] K. Pahlavan and A. H. Levesque, "Wireless data communications," Proc. IEEE, vol. 82, pp. 1398-1430, Sept. 1994.

[18] L. Scharf and B. D. Van Veen, "Low rank detectors for Gaussian random vectors," IEEE Trans. Acoust., Speech, Signal Processing, pp. 1579-1582, Nov. 1987.

[19] B. D. Van Veen, "An analysis of several partially adaptive beamformer designs," IEEE Trans. Acoust., Speech, Signal Processing, pp. 192-203, Feb. 1989.

[20] W. C. Jakes, "Mobile radio propagation," in Microwave Mobile Communications, W. C. Jakes, Ed. New York: Wiley, pp. 11-78, 1974.

[21] A. M. Sayeed and B. Aazhang, "Communication over multipath fading channels: A time-frequency perspective," in Wireless Communications: TDMA versus $C D M A$, S. G. Glisic and P. A. Lippänen, Eds. Norwell, MA: Kluwer, 1997, pp. 73-98.

[22] G. W. Wornell, "Spread-signature CDMA: Efficient multiuser communications in the presence of fading," IEEE Trans. Inform. Theory, vol. 41, pp. 1418-1438, Sept. 1995.

[23] R. A. Iltis and A. W. Fuxjaeger, "A digital DS spread-spectrum receiver with joint channel and Doppler shift estimation," IEEE Trans. Commun., vol. 39, pp. 1255-1267, Aug. 1991.

[24] A. W. Fuxjaeger and R. A. Iltis, "Adaptive parameter estimation using parallel Kalman filtering for spread spectrum code and Doppler tracking," IEEE Trans. Commun., vol. 42, pp. 2227-2230, June 1994. 
[25] D. Brady and J. Catipovic, "Adaptive multiuser detection for underwater acoustical channels," IEEE J. Ocean. Eng., vol. 19, pp. 158-165, Apr 1994.

[26] E. Sourour and M. Nakagawa, "Performance of orthogonal multicarrier CDMA in a multipath fading channel," IEEE Trans. Commun., vol. 44, pp. 356-367, Mar. 1996.

[27] A. M. Sayeed and B. Aazhang, "Multiuser timing acquisition over multipath fading channels," in Proc. 1998 Conf. Information Science Systems (CISS'98).

[28] A. M. Sayeed, A. Sendonaris, and B. Aazhang, "Multiuser detectors for fast-fading multipath channels," in Proc. 31st Asilomar Conf. Signals, Systems, and Computers, 1997, pp. 603-608.

[29] - "Multiuser detection in fast fading multipath environments," IEEE J Select. Areas Commun., to be published.

[30] R. A. Iltis, "Joint estimation of PN code delay and multipath using the extended Kalman filter," IEEE Trans. Commun., vol. 38, pp. 1677-1685, Oct. 1990.

[31] M. K. Tsatsanis, G. B. Giannakis, and G. Zhou, "Estimation and equalization of fading channels with random coefficients," Signal Processing, vol. 53, pp. 211-229, 1996.

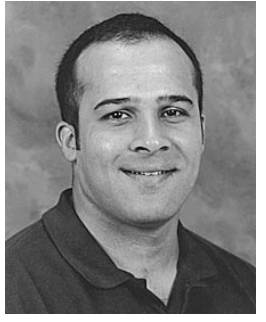

Akbar M. Sayeed (S'87-M'97) received the B.S. degree from the University of Wisconsin-Madison in 1991 and the M.S. and Ph.D. degrees in 1993 and 1996, respectively, from the University of Illinois at Urbana-Champaign, all in electrical engineering.

While at the University of Illinois, he was a Research Assistant with the Coordinated Science Laboratory and the Schlumberger Fellow in signal processing. During 1996-1997, he was a PostDoctoral Fellow at Rice University, Houston, TX Since August 1997, he has been with the University of Wisconsin-Madison, where he is currently an Assistant Professor. His research interests are in signal processing for wireless communications, statistical- and time-varying signal processing and time-frequency, and wavelet analysis.

Behnaam Aazhang (S'82-M'85-SM'98), photograph and biography not available at the time of publication. 